\title{
Bacterial Microbiomes Associated with the Rhizosphere, Root Interior, and Aboveground Plant Organs of Wheat and Canola at Different Growth Stages
}

\author{
Jorge Cordero Elvia, ${ }^{\dagger}$ J. Renato de Freitas, and James J. Germida \\ Department of Soil Science, University of Saskatchewan, 51 Campus Drive, Saskatoon, SK S7N 5A8, Canada
}

Accepted for publication 2 May 2021.

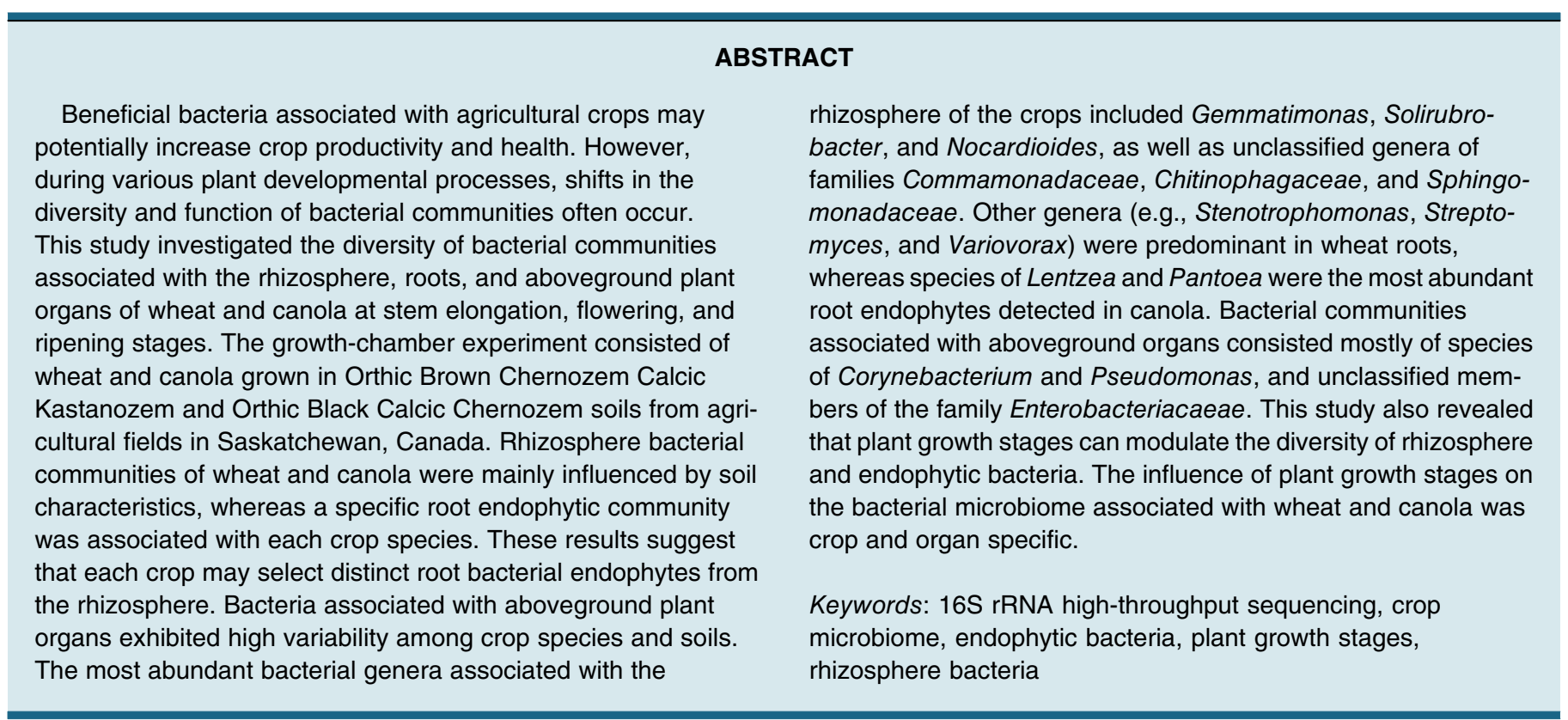

The microbiome associated with plants includes all microorganisms inhabiting external surfaces and internal plant organs. Due to their relevance to plant growth and development, the microbiome is sometimes considered the plant's second genome (Turner et al. 2013). Among these microorganisms, bacteria are important in promoting plant growth and health, or improving the sustainability of crop production (Berg et al. 2014). For example, crop-associated bacteria have the potential to stimulate plant growth by several mechanisms, including (i) production of plant growth regulators, (ii) amelioration of abiotic stress, (iii) biological nitrogen fixation, and

${ }^{\dagger}$ Corresponding author: J. Cordero Elvia; joc238@mail.usask.ca

*The $e$-Xtra logo stands for "electronic extra" and indicates that supplementary materials and supplementary figures are published online.

The author(s) declare no conflict of interest.

(C) 2021 The American Phytopathological Society (iv) phosphorus mobilization (Ali et al. 2014; Compant et al. 2010). In addition, bacteria may share habitats similar to those of phytopathogens, thus making it possible to limit the spread of potential pathogens (Reinhold-Hurek and Hurek 2011).

Bacterial communities colonize plant tissues mainly using two pathways, including horizontally (i.e., entering the plant tissues from the environment) or vertically (i.e., transmitted from the parent plant to its offspring through seed) (Bright and Bulgheresi 2010). Bacterial colonization of crops begins at seed germination, in which seed exudates attract bacteria from soil into the spermosphere (Nelson 2004). Additionally, during further crop growth, plant root exudates also are important in shaping the microbiome in the rhizosphere, the rhizoplane, and, subsequently, the root interior. After entering roots, bacterial endophytes may translocate into the xylem vessels and eventually colonize the aboveground plant tissues (Kandel et al. 2017). Bacteria may also colonize aboveground surfaces and penetrate plant organs such as stem, leaves, flowers, and fruit. Potential sources of bacteria for aboveground plant colonization include the atmosphere, rain, or pollinators (Frank et al. 2017). In addition, 
vertical transmission of bacteria through seed has been described as the main pathway for the colonization of most obligate endophytes, which depend strictly on the host plant for their growth and survival (Hardoim et al. 2008). Several studies have reported the diversity of bacteria colonizing aboveground tissues such as stems, leaves, and seed (Coombs and Franco 2003; Hardoim et al. 2012; Sessitsch et al. 2002).

Previous studies by el Zahar Haichar et al. (2008), Germida et al. (1998), Lay et al. (2018), and Ofek-Lalzar et al. (2014) reported that bacterial communities associated with crops are strongly influenced by plant species or cultivar. For example, the selection of rhizosphere microbiota from the bulk soil is modulated by the release of root exudates by the crops (Bulgarelli et al. 2013). Bacterial penetration into the root interior is also influenced by the morphology of the root system because bacterial colonization occurs mainly by accessing root tips or epidermis discontinuities (e.g., lateral root emergence sites or wounds) (Gaiero et al. 2013). Bacterial multiplication within internal plant tissues also involves the recognition of plant signals that may induce cellular processes necessary for the establishment of endophytes inside the host crop (Hardoim et al. 2008). Aerial vegetative organs are also colonized by bacterial endophytes adapted to specific plant niches in the aboveground plant organs (Hallmann 2001). Among plant characteristics, the physiological processes occurring during plant growth may influence the bacterial microbiome of crops. Previous studies report that rhizosphere bacterial communities associated with Arabidopsis, Medicago, maize, pea, wheat, canola, and sugar beet change during plant developmental stages (Baudoin et al. 2002; Dunfield and Germida 2003; Houlden et al. 2008; Micallef et al. 2009; Mougel et al. 2006). However, studies analyzing the influence of plant development stages on the bacterial microbiome associated with both below- and aboveground plant compartments are scarce.

Canola and wheat production are important agronomical activities and food exports in Canada, accounting for 19 and 26 million metric tons, respectively, in 2020 (Statistics Canada 2021). Despite their relevance in Canadian agriculture, research about the microbiomes of canola and wheat is still scarce. Our recent investigation (Cordero et al. 2020) indicated that field-grown canola, wheat, field pea, and lentil collected at the flowering stage supported distinct root endophytic bacterial communities, suggesting that crop species strongly influence the selection of root bacterial microbiomes. Furthermore, we found that rhizosphere communities exhibited distinct profiles among crops and sampling locations. These results raised questions about how soil characteristics, plant growth stage, and plant compartments influenced bacterial microbiomes. In the present study, we assessed the diversity of bacteria colonizing the rhizosphere, roots, shoots, and seed of wheat (Triticum aestivum L.) and canola (Brassica napus L.) plants at stem elongation, flowering, and ripening stages and grown on soils collected at Central Butte and Melfort, Saskatchewan, Canada. Our hypothesis was that plant compartment, plant growth stages, and soil characteristics affect the bacterial microbiomes of wheat and canola. The study was performed under controlled plant growth conditions, excluding effects of environmental conditions and agronomical practices existing between sampling locations.

\section{MATERIALS AND METHODS}

Experimental design. Orthic Brown Chernozem Calcic Kastanozem (hereafter referred to as brown) and Orthic Black Calcic Chernozem (hereafter referred to as black) soils were collected from wheat fields at Central Butte $\left(50^{\circ} 43^{\prime} \mathrm{N}, 106^{\circ} 25^{\prime} \mathrm{W}\right)$ and Melfort $\left(52^{\circ} 49^{\prime} \mathrm{N}\right.$, $\left.104^{\circ} 36^{\prime} \mathrm{W}\right)$, Saskatchewan, Canada, respectively, which differed mainly in soil texture, organic matter content, and $\mathrm{pH}$. These sites were adjacent to our previous field study (Cordero et al. 2020).
Bulk soil was air dried and sieved $(<2 \mathrm{~mm})$ and properties were determined by ALS Environmental Laboratory (Saskatoon, Saskatchewan, Canada). The brown soil was characterized as a sandy loam with $\mathrm{pH}=$ 7.6; organic matter $(\%)=3.1$; and available nutrients $\mathrm{N}^{-\mathrm{NO}_{3}}{ }^{-}=5.5$, $\mathrm{S}_{-} \mathrm{SO}_{4}{ }^{2-}=6.6, \mathrm{P}_{-} \mathrm{PO}_{4}{ }^{3-}=28.6$, and $\mathrm{K}^{+}=584 \mathrm{mg} \cdot \mathrm{g}^{-1}$ of soil; whereas the black soil was a silty clay loam with $\mathrm{pH}=6.1$; organic matter $(\%)=14.6$; and available nutrients $\mathrm{N}_{-} \mathrm{NO}_{3}{ }^{-}=15.9, \mathrm{~S}_{-} \mathrm{SO}_{4}{ }^{2-}=7.1$,

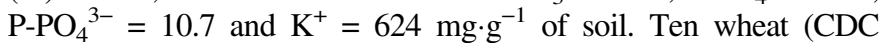
Waskeda) or canola (Invigor L150) seeds were seeded in 2.5-liter pots $(n=4)$ using $1.5 \mathrm{~kg}$ of soil, allowed to germinate, thinned to two plants, and grown in a growth chamber with a cycle of $16 \mathrm{~h}$ at $25^{\circ} \mathrm{C}$ (day, $1,500 \mu \mathrm{mol} \cdot \mathrm{m}^{-2}$ ) and $8 \mathrm{~h}$ at $15^{\circ} \mathrm{C}$ (night). For each crop and soil, three individual sets of pots were prepared to be harvested independently at stem elongation, flowering, and ripening stages following a universal plant growth stage scale (Lancashire et al. 1991). Pots were arranged in a fully randomized block design with four replicates for a total of 48 pots. Plants were watered with autoclaved distilled water at 50\% moisture-holding capacity throughout the experiment and rotated daily. To ensure an adequate fertilization regime, 1:5 Hoagland's nutrient solution was added to each pots on alternate weeks until plants reached the flowering stage.

Sampling of rhizosphere soil, root, stem, leaf, and seed. In total, 24 wheat and 24 canola plants were harvested from black and brown soils at stem elongation, flowering, and ripening. At each sampling time, a set of canola and wheat plants grown at brown and black soils were harvested $(n=16)$. After collecting plants, the bulk soil was manually removed from roots and the aboveground plant organs were separated from the roots using aseptic techniques. Plant roots (two roots/pot) with adhering soil were placed in a 500-ml Erlenmeyer flask containing $200 \mathrm{ml}$ of sterile phosphate-buffered saline (PBS) and placed on a rotary shaker $(150 \mathrm{rpm})$ at $22^{\circ} \mathrm{C}$ for $25 \mathrm{~min}$. The resulting soil slurry was transferred to $50-\mathrm{ml}$ Falcon tubes and centrifuged $(2,000 \times g$ for $5 \mathrm{~min})$. The supernatant containing PBS buffer was discarded and the rhizosphere soil was stored at $-80^{\circ} \mathrm{C}$ for DNA extraction (Dunfield and Germida 2003). Roots, stems, or leaves were washed with sterile tap water to eliminate soil and particles from the plant surface. Next, washed plant material (from a single plant) was surface disinfested using a 300-ml Erlenmeyer flask containing $100 \mathrm{ml} \mathrm{NaClO}\left(1.05 \% \mathrm{vol} \mathrm{vol}^{-1}\right)$ in autoclaved PBS. The flasks were placed on a rotary shaker $(150 \mathrm{rpm})$ at $22^{\circ} \mathrm{C}$ for $15 \mathrm{~min}$ (Siciliano and Germida 1999). Next, the surface-disinfected plant material was cut into $0.5-\mathrm{cm}$ portions using a sterile scalpel and stored at $-80^{\circ} \mathrm{C}$ until processed for molecular analyses. Furthermore, surface disinfection of collected seed was accomplished by submerging the seed in $65 \%\left(\mathrm{vol} \mathrm{vol}^{-1}\right)$ ethanol for $3 \mathrm{~min}$, followed by submersion in $\mathrm{NaClO}\left(1.2 \% \mathrm{vol} \mathrm{vol}^{-1}\right.$ ) for $5 \mathrm{~min}$ (Vincent 1970). After the disinfection process, plant material was rinsed 10 times in sterile tap water and efficacy of surface sterilization was checked by spreading $0.1 \mathrm{ml}$ of the last washes onto 1/10th strength tryptone soy solidified with $1.5 \%$ agar (Siciliano and Germida 1999). Rhizosphere, root, and stem were collected at each growth stage. Leaves were collected only at stem elongation and flowering, whereas seed were collected only at ripening. In total, 96 wheat and 96 canola samples were obtained from the rhizosphere, root, stem, leaf, and seed.

Analysis of bacterial communities using 16S ribosomal RNA high-throughput sequencing. Total genomic DNA was extracted from rhizosphere soil and plant material using soil and plant DNA extraction kits, respectively (MO BIO Laboratories, Inc.). The DNA extractions were conducted following the manufacturer's protocols. The DNA yield was quantified using a Qubit DNA HS Assay Kit (Thermo Fisher Scientific). DNA visualization was performed after running DNA electrophoresis in $1 \%$ agarose gels stained with the SYBR safe DNA gel stain (Invitrogen) by comparison with a DNA mass ladder (Invitrogen) using a Bio-Rad Gel Doc XR System 
(Bio-Rad Laboratories, Mississauga, ON, Canada). The DNA samples were submitted for high-throughput sequencing to the Génome Québec Innovation Centre, McGill University, using Illumina MiSeq PE250 technology. The PCRs were conducted using the primers 520F (5'-AGCAGCCGCGGTAAT-3') and 799R2 (5'-CAGGGTATCT AATCCTGTT- $3^{\prime}$ ) that amplified the V4 region of the $16 \mathrm{~S}$ ribosomal RNA (rRNA) gene (Edwards et al. 2007). Sample libraries were prepared according to the MiSeq reagent kit preparation guide (Illumina, San Diego, CA, U.S.A.), and the sequencing protocol from Caporaso et al. (2010).

Bioinformatics and statistical analyses. The DNA sequences derived from rhizosphere and plant endophytic bacteria using highthroughput Illumina technology were analyzed using Mothur v.1.34.3 (Kozich et al. 2013). The standard operating procedure included the combination of forward and reverse reads and removal of sequence errors and chimeras. Taxonomic classification was performed with naive Bayesian classifier using the SILVA database (Silva.seed_v128). During sequence processing, 16S rRNA sequences identified as archaea, plant plastid, and mitochondria were detected. For both crops, the removed chloroplast sequences accounted for $<0.01 \%$ in the rhizosphere, $0.02 \%$ in the root, $1.5 \%$ in the stem, $3.3 \%$ in the leaf, and $1.2 \%$ in the seed. The removed mitochondrial sequences accounted for $0.05 \%$ in the rhizosphere, $17 \%$ in the root, $87 \%$ in the stem, $84 \%$ in the leaf, and $95 \%$ in the seed (Supplementary Fig. S1). These sequences were removed from the dataset before further microbiome analysis. Reads displaying at least $97 \%$ identity were clustered into operational taxonomic units (OTU). Relative abundance of individual bacterial taxa in a sample was calculated as the percentage of sequence reads belonging to the bacterial taxa in relation to the total number of reads in a sample. Principal coordinate analysis (PCoA) based on Bray-Curtis distances was performed using QIIME (Quantitative Insights Into Microbial Ecology 1.9.1) (Caporaso et al. 2010) to visualize the bacterial community structure of rhizosphere, root interior, and aboveground organs. The influence of crop, soil, plant compartment, and development stages on the bacterial communities was examined by permutational multivariate analysis of variance (PERMANOVA) of the OTU profiles using PCOrd software (McCune and Grace 2002). Heatmaps of the most abundant genera (relative abundance higher than $0.5 \%$ ) and the calculation of Simpson's reciprocal (1/D) diversity and Chao 1 richness were conducted using the VEGAN package (version 2.0-7) in R, version 2.15.2 (R Core Team 2021). Differential abundance analyses was used to detect OTUs that showed significantly differential relative abundance between plant compartments, crop species, soil types, and plant growth stages. Differential abundance analyses were conducted using the DESeq2 package in $\mathrm{R}$, version 4.05 (R Core Team 2021) with the Wald statistical test (Love et al. 2014). $\log _{2}$ fold-changes and adjusted $P$ values were reported for each comparison. The top 10 OTUs with adjusted $P$ values $<0.05$ were considered to be significantly different in a differential comparison. The richness and diversity indexes were subjected to analysis of variance and Tukey post hoc test using SAS software, version 9.6 (SAS Institute Inc., Cary, NC, U.S.A.). The sequence data can be accessed in NCBI under Genome Project ID 510722 (accession PRJNA510722).

\section{RESULTS}

Community structure and diversity of bacterial communities in plant compartments. The $16 \mathrm{~S}$ rRNA gene sequencing of all samples from wheat and canola rhizosphere and plant organs at stem elongation, flowering and ripening stages generated a total of 3,769,710 high-quality reads. Sequence processing identified 9,857 bacterial OTUs at $97 \%$ similarity across all samples, corresponding to 8,453

OTUs and 8,984 OTUs in wheat and canola, respectively (Table 1). The rhizocompartments (rhizosphere and roots) in both crops exhibited the highest number of OTUs, followed by stems and leaves, with seed exhibiting the lowest values (Table 1). Venn diagrams representing the OTU distribution revealed that 7,580 OTUs were shared between wheat and canola, which represent more than $80 \%$ of the bacterial OTUs of both crops (Fig. 1). Plants grown in black and brown soils shared 2,850 and 3,270 OTUs in wheat and canola, respectively, which represent more than $40 \%$ of the bacterial microbiome of each crop. The rhizosphere, the root interior, and the aboveground plant organs shared 359 and 449 OTUs in wheat and canola, respectively. In both crops, the number of OTUs shared between the rhizosphere and aboveground plant organs was higher than OTUs shared between the root interior and aboveground plant organs (Fig. 1). In addition, the number of OTUs shared between the rhizosphere and root interior was higher in comparison with the number of OTUs shared among other plant compartments.

In wheat and canola, the Chao 1 richness estimator and Simpson's $1 / \mathrm{D}$ values were influenced $(P<0.0001)$ by soils and plant compartment, as well as the interaction soil-compartment (Supplementary

TABLE 1

Number of bacterial sequences and operational taxonomic units (OTUs) in wheat and canola grown in brown and black soils from Saskatchewan ${ }^{a}$

\begin{tabular}{|c|c|c|c|c|}
\hline Crop, soil & Compartment & Samples & Sequences & OTUs \\
\hline \multicolumn{5}{|l|}{ Wheat } \\
\hline \multirow[t]{5}{*}{ Brown } & Rhizosphere & 12 & 474,997 & 5,906 \\
\hline & Root & 12 & 190,596 & 533 \\
\hline & Stem & 12 & 22,664 & 298 \\
\hline & Leaf & 8 & 24,307 & 182 \\
\hline & Seed & 4 & 4,966 & 114 \\
\hline \multirow[t]{5}{*}{ Black } & Rhizosphere & 12 & 429,340 & 5,032 \\
\hline & Root & 12 & 285,028 & 819 \\
\hline & Stem & 12 & 13,322 & 291 \\
\hline & Leaf & 8 & 4,521 & 234 \\
\hline & Seed & 4 & 482 & 101 \\
\hline Total & - & 96 & $1,450,223$ & 8,453 \\
\hline \multicolumn{5}{|l|}{ Canola } \\
\hline \multirow[t]{5}{*}{ Brown } & Rhizosphere & 12 & 480,725 & 6,096 \\
\hline & Root & 12 & 610,845 & 1,431 \\
\hline & Stem & 12 & 34,383 & 320 \\
\hline & Leaf & 8 & 65,132 & 172 \\
\hline & Seed & 4 & 1,754 & 103 \\
\hline \multirow[t]{5}{*}{ Black } & Rhizosphere & 12 & 461,826 & 5,107 \\
\hline & Root & 12 & 549,861 & 1,328 \\
\hline & Stem & 12 & 73,477 & 470 \\
\hline & Leaf & 8 & 39,125 & 161 \\
\hline & Seed & 4 & 2,359 & 85 \\
\hline Total & - & 96 & $2,319,487$ & 8,984 \\
\hline Grand total & - & 192 & $3,769,710$ & 9,857 \\
\hline
\end{tabular}


Table S1). In both crops, the highest richness was detected in the rhizosphere, followed by root, whereas no significant differences were detected between stem, leaf, and seed (Supplementary Tables S2 and S3). In addition, the rhizosphere of both crops exhibited a higher bacterial richness in plants grown in the brown soil when compared with black soil. However, no differences were observed when comparing bacterial richness in the roots, stems, leaves, and seed of plants grown on different soils. The bacterial diversity (Simpson's index) in both crops grown in brown or black soils was higher in the rhizosphere, whereas no significant differences were detected between root, stem, leaf, and seed (Supplementary Tables S2 and S3).

Plant growth stages, as well as soil-stage and compartment-stage interactions, influenced $(P<0.05)$ bacterial Chao 1 richness and Simpson 1/D diversity in wheat (Supplementary Table S1). The rhizosphere and roots of wheat plants grown in black soils exhibited higher richness and diversity at the flowering stage (Supplementary Table S2). A similar trend was noted for the bacterial richness in the roots and stems of wheat plants grown in brown soils. In canola, only bacterial richness was affected $(P<0.05)$ by plant growth stages and the compartment-stage interaction, whereas diversity was not influenced (Supplementary Table S1). The stems of canola plants grown in brown and black soils exhibited higher richness at stem elongation and flowering stage, respectively (Supplementary Table $\mathrm{S} 3$ ). The bacterial richness was also higher at flowering in the roots and rhizosphere of wheat grown in brown and black soils, respectively (Supplementary Table S3).

PCoA revealed distinct patterns among the rhizosphere, root, and aboveground bacterial communities associated with the crops, with the first two axes explaining $33 \%$ of the total variation of the bacterial communities (Supplementary Fig. S2). Bacterial patterns of the rhizosphere and roots exhibited individual clusters in the ordination. However, bacterial profiles associated with the stem, leaf, and seed greatly overlapped. PERMANOVA determined that bacterial community structure differed between the plant compartments $(P \leq 0.001)$ (Supplementary Table S4). PCoA of bacteria associated with the rhizocompartments indicated that rhizosphere bacterial communities of plants grown on black and brown soils separated along axis 1 (Fig. 2). Root bacterial communities of canola and wheat plants greatly separated along axis 1 , whereas wheat communities also separated along axis 2, corresponding with each soil type (Fig. 2). Although clustering was observed based on plant species and soils, no clusters were observed among plant growth stages in the rhizocompartments (Fig. 2; Table 2). PERMANOVA confirmed that root endophytic communities were influenced by soil or crop species as well as the crop-soil interaction, whereas rhizosphere communities were only influenced by soil type (Table 2 ).

The PCoA of the bacteria associated with the aboveground plant organs revealed that bacterial patterns in the stem exhibited a cluster of samples at ripening stage, whereas no clustering was detected at stem elongation or maturity (Fig. 2). PERMANOVA confirmed that endophytic bacterial communities associated with the stem were highly influenced $(P<0.001)$ by plant growth stage, and crop and plant species also affected $(P<0.01)$ bacterial stem profiles (Table 2). Bacterial communities associated with the stem exhibited no clustering among samples in response to soils, crop species, or plant growth stages (Fig. 2). However, PERMANOVA indicated that crops species and plant growth stages influenced $(P<0.01)$ bacterial profiles (Table 2). Seed bacterial communities were not influenced by soils or crops (Fig. 2; Table 2).

Taxonomic analysis of bacterial communities in the plant compartments. Taxonomic classification of the bacterial OTUs from potted-soil-grown wheat and canola revealed 13 phyla. Overall, nine bacterial phyla were observed concomitantly in wheat and canola grown in brown and black soils (Supplementary Fig. S3). Proteobacteria, Actinobacteria, Gemmatimonadetes, Bacteroidetes, and Firmicutes were the dominant phyla in both wheat and canola plants.

Differential abundance analysis revealed the top 10 bacterial OTUs that exhibited higher significant differences $(P<0.05)$ in relative abundance among plant compartments, crop species, and soil types. However, OTUs did not exhibit significant differences among plant
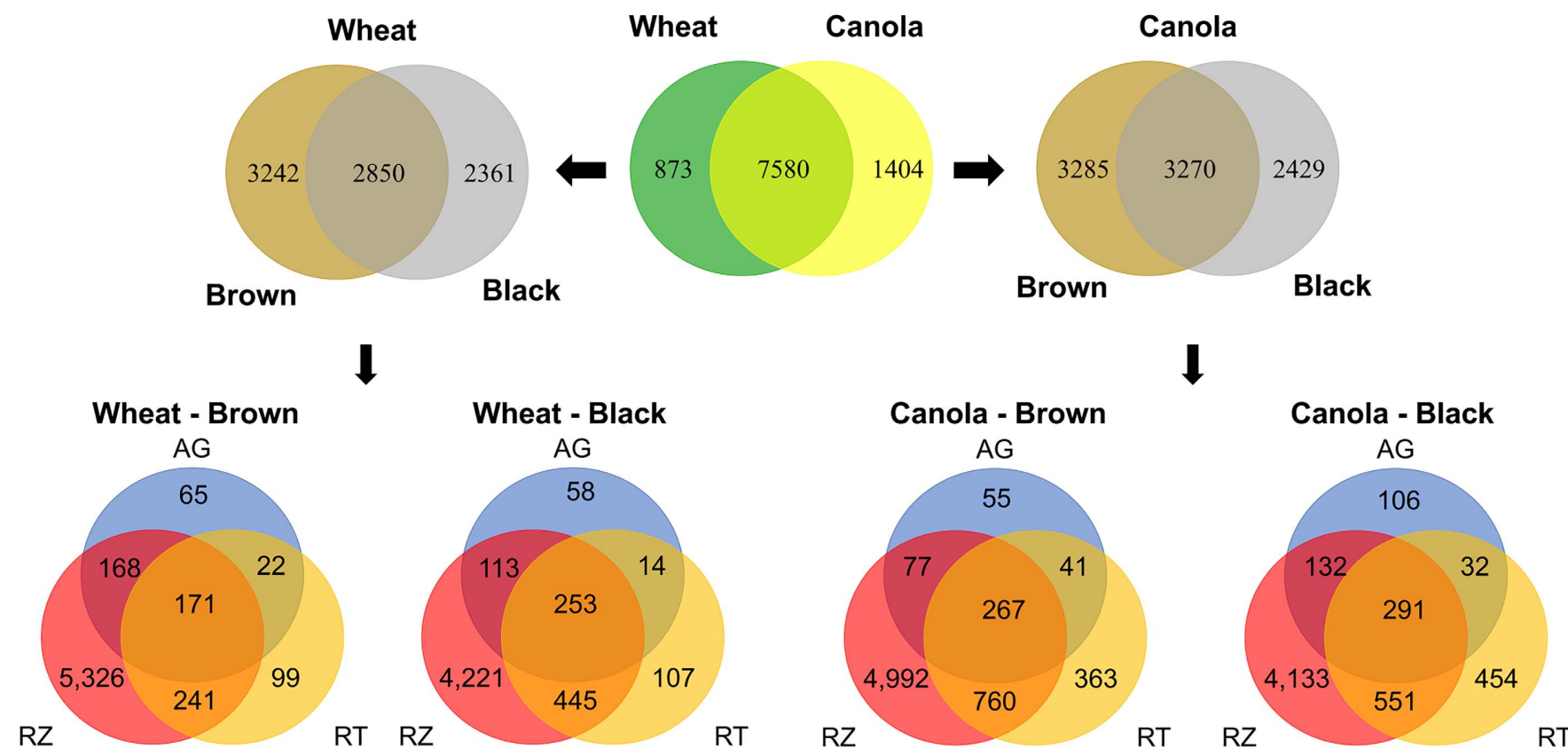

Fig. 1. Venn diagram representing the bacterial operational taxonomic units associated with the rhizosphere (RZ), root (RT), and aboveground plant organs (AG) of wheat and canola grown in an Orthic Brown Chernozem Calcic Kastanozem and Orthic Black Calcic Chernozem soils from Central Butte and Melfort, Saskatchewan, respectively. Data presented were obtained from plants harvested at stem elongation, flowering, and ripening. 
growth stages (Supplementary Table S5). Relative abundance of the genera Cellvibrio and Vasilyevaea was higher in the rhizosphere, whereas Cupriavidus and Chitinophaga were predominant in the roots (Supplementary Fig. S4). The genera Paenibacillus and Arthrobacter were the most abundant bacterial taxa in the stems, whereas leaf and seed exhibited a low abundance of the detected differential OTUs. Differential analysis between crop species revealed that the genera Moraxella and Methylophilus were predominant in canola whereas, in wheat, Stenotrophomonas, Asticcacaulis, and Sphingobium were the most abundant genera (Supplementary Fig. S4). Most of bacterial taxa detected as differential OTUs among soils were highly abundant in plants grown on brown soil; however, Amycolatopsis and Porphyrobacter were predominant in plants grown black soils (Supplementary Fig. S4).

The most abundant bacterial genera associated with the rhizosphere and root interior of wheat were grouped into four main clusters: rhizosphere of wheat grown in brown soil (cluster A) and in black soil (cluster B), and root interior of wheat grown in black soil (cluster C) and in brown soil (cluster D) (Supplementary Fig. S5). The most prevalent genera in the rhizosphere of wheat grown in brown soil
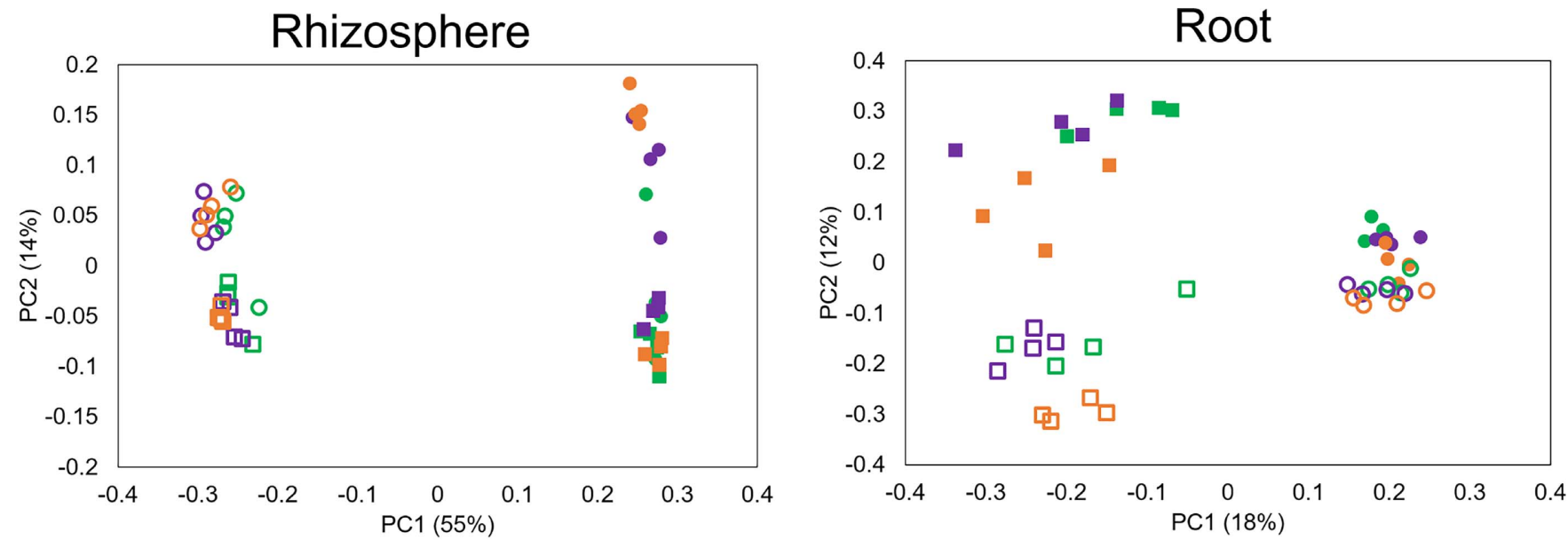

Stem
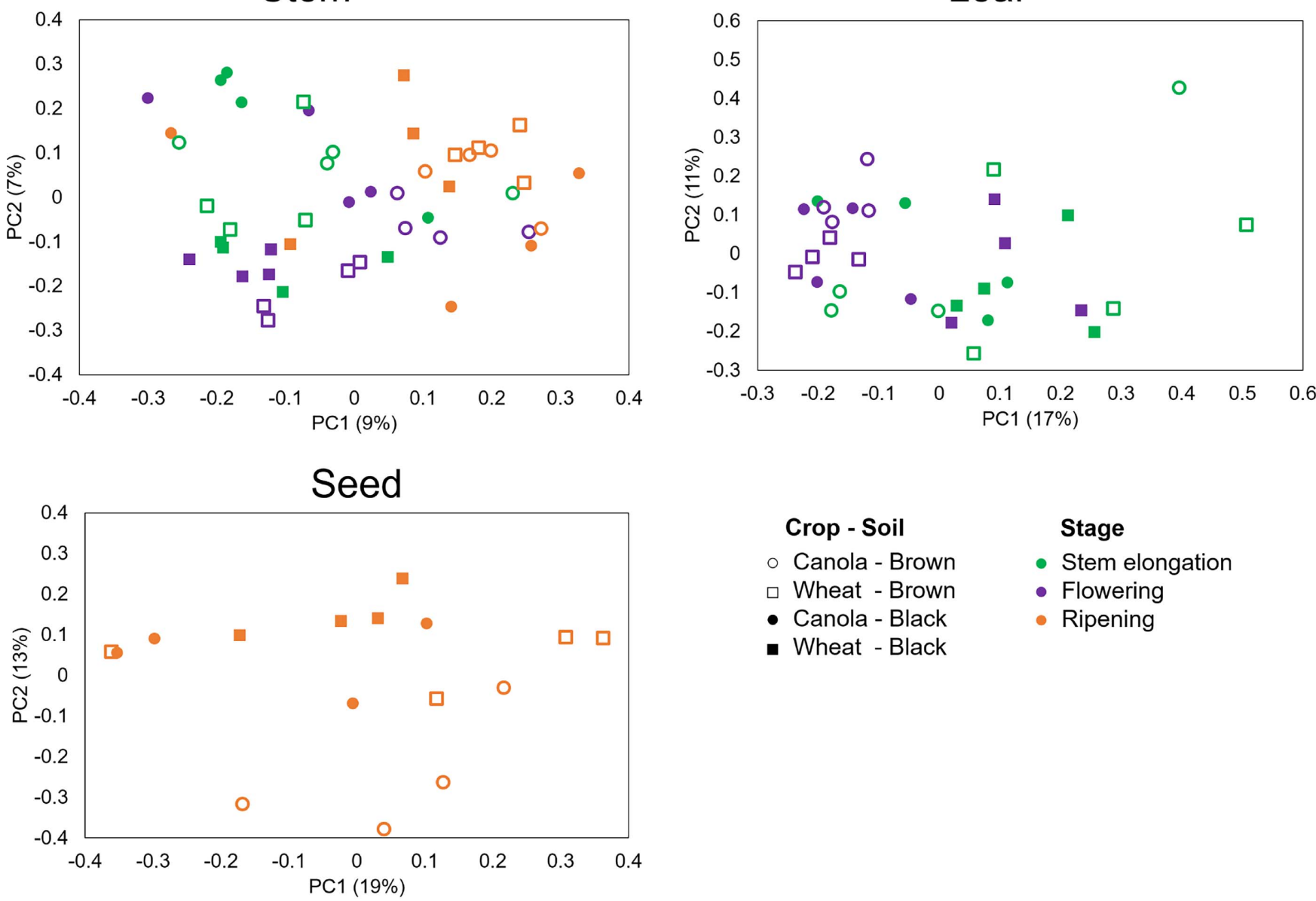

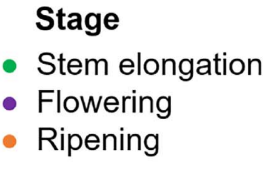

Stage

- Canola - Brown

- Stem elongation

- Wheat - Brown

- Canola - Black

- Wheat - Black

Fig. 2. Principal coordinate (PC) analysis based on Bray-Curtis dissimilarity between bacterial communities associated with wheat and canola grown in brown and black soils from Saskatchewan. 
(cluster A) were Lysobacter (3\%), Sphingomonas (3\%), and unclassified genera of the families Burkholderiales (4\%), Comamonadaceae (4\%), Chitinophagaceae (4\%), Rhizobiales (4\%), and Sphingomonadaceae $(3 \%)$. In addition, the rhizosphere of wheat grown in black soil (cluster B) was dominated by Arthrobacter (4\%), Gemmatimonas (3\%), Nocardiodes (4\%), Solirubrobacter (5\%), and unclassified genera of the families Actinomycetales (3\%), Geodermatophilaceae (4\%), Rhizobiales (5\%), and Solirubrobacterales (7\%). In contrast, the root interior of wheat grown in black soil (cluster C) exhibited a high abundance of Chitinophaga (7\%), Lysobacter (4\%), Streptomyces (10\%), Variovorax (4\%), and unclassified genera of the families Enterobacteriaceae (33\%), and Oxalobacteraceae (4\%). However, the bacterial communities associated the root interior of wheat grown in brown soil (cluster D) mainly consisted of Variovorax (10\%), Devosia (3\%), and unclassified genera of the families Comamonadaceae (3\%) Enterobacteriaceae (3\%), and Pseudonocardiaceae (6\%). These findings suggest that rhizosphere communities of wheat and canola were influenced by soil characteristics.

The most abundant bacterial genera associated with the rhizosphere and root interior of canola were grouped in three main clusters: root interior of canola grown in brown and black soils (cluster A), and rhizosphere of canola grown in brown soil (cluster B) and in black soil (cluster C) (Supplementary Fig. S6). Root samples (cluster A) were characterized by the prevalence of the genera Streptomyces (3\%), Variovorax (3\%), and unclassified genera of the families Enterobacteriaceae (38\%) and Pseudonocardiaceae $(11 \%)$. Additionally, the dominant genera in the rhizosphere of canola grown in brown soil (cluster B) were Sphingomonas $(3 \%)$ and unclassified genera of the families Burkholderiales (7\%), Comamonadaceae (4\%), Chitinophagaceae (4\%), Enterobacteriaceae (3\%) Rhizobiales (3\%), and Sphingomonadaceae (3\%). In contrast, Gemmatimonas (5\%), Solirubrobacter (4\%), Lysobacter $(4 \%)$, Variovorax (4\%), Sphingomonas (3\%), Nocardiodes $(3 \%)$, and unclassified genera of the families Geodermatophilaceae (3\%), Rhizobiales (3\%), Solirubrobacterales (5\%), and Xanthomonadaceae $(5 \%)$ were dominant in the rhizosphere of canola grown in black soil (cluster $\mathrm{C}$ ). These results suggest during endophytic colonization, each crop may select distinct bacterial communities.

The distribution of the bacterial genera associated with the aboveground plant organs in both crops was highly variable among soils and growth stages; thus, no clusters were detected in response to these factors (Supplementary Figs. S7 and S8). Overall, the relative abundance of unclassified genera of the family Enterobacteriacaeae was high in the aboveground plant samples and accounted for up to 99 and $97 \%$ of bacterial communities in wheat and canola, respectively (Supplementary Figs. S7 and S8). Similarly, wheat and canola aboveground plant organs also exhibited a high abundance of genera Corynebacterium and Pseudomonas that accounted for up to 40 and $37 \%$ of the bacterial profile, respectively. In addition, Tumebacillus (2\%) and unclassified genera of the family Planococcaceae (2\%) were also detected in wheat aboveground plant organs (Supplementary Fig. S7). Conversely, Acinetobacter (2\%), Staphylococcus $(2 \%)$, and unclassified genera of the order Actinomycetales $(2 \%)$ were also detected in canola plants (Supplementary Fig. S8).

Influence of plant development stages on bacterial microbiome. Bacterial communities associated with the rhizosphere, roots, and stems of canola and wheat were analyzed for their bacterial profiles at different plant growth stages (Figs. 3 and 4). The leaves and seed of both crops were excluded from this analysis due to their absence during the plant development stages assessed. In this study, the predominance of the most abundant bacterial families at stem elongation, flowering, and ripening was influenced by crop species and plant compartments. For example, the bacterial profile in the rhizosphere exhibited an even distribution of the relative abundance of most families among growth stages in both crops. However, the family Enterobacteriaceae was higher in the rhizosphere of canola at ripening when compared with stem elongation and flowering (Figs. 3 and 4).

The analyzes of roots indicated that the relative abundance of bacterial families in canola exhibited an even distribution among various plant growth stages (Fig. 4). In contrast, wheat roots exhibited a more variable bacterial distribution among plant growth stages. For example, at stem elongation stage, families Bacillaceae, Geodermatophilaceae, Gemmatimonadaceae, Planococcaceae, and Solirubrobacteraceae were highly abundant in wheat roots, whereas the family Oxalobacteraceae was predominant at flowering stage. Additionally, at ripening, a high abundance of the families Mycobacteriaceae, Pseudonocardiaceae, Streptomycetaceae, Enterobacteriaceae, Comamonadaceae, Hyphomicrobiaceae, Rhizobiaceae, and Bradyrhizobiaceae was detected (Fig. 3).

The distribution of bacterial families varied greatly among plant growth stages of wheat and canola stems (Figs. 3 and 4). For example, in wheat, most of bacterial families were scarce or absent at stem elongation. In contrast, the families Geodermatophilaceae, Pseudonocardiaceae, Solirubrobacteraceae, Streptomycetaceae, and Enterobacteriaceae were dominant in wheat stems at flowering. At ripening, the families Chitinophagaceae, Sphingobacteriaceae, Alicyclobacillaceae, Bacillaceae, Planococcaceae, Nocardioidaceae, and Bradyrhizobiaceae were also prevalent in wheat stems (Fig. 3). Conversely, in canola stems, the relative abundance of most families was higher at stem elongation and flowering stages, except that families Chitinophagaceae and Sphingobacteriaceae were highly prevalent at ripening (Fig. 4).

TABLE 2

Effect of crop species, soil type, and plant growth stage on bacterial operational taxonomic unit community composition assessed with permutational multivariate analysis of variance ${ }^{a}$

\begin{tabular}{|c|c|c|c|c|c|c|c|c|c|c|c|}
\hline \multirow[b]{2}{*}{ Variable } & \multirow[b]{2}{*}{$\mathrm{dF}$} & \multicolumn{2}{|c|}{ Rhizosphere } & \multicolumn{2}{|c|}{ Root } & \multicolumn{2}{|c|}{ Stem } & \multicolumn{2}{|c|}{ Leaf } & \multicolumn{2}{|c|}{ Seed } \\
\hline & & $F$ & $P$ & $F$ & $P$ & $F$ & $P$ & $F$ & $P$ & $F$ & $P$ \\
\hline Crop & 1 & 1.7 & $0.15 \mathrm{~ns}$ & 9.7 & $0.0002^{* * *}$ & 1.7 & $0.001^{* *}$ & 2.2 & $0.003^{*}$ & 1.2 & $0.19 \mathrm{~ns}$ \\
\hline Stage & 2 & 0.8 & $0.62 \mathrm{~ns}$ & 1.3 & $0.07 \mathrm{~ns}$ & 1.8 & $0.0002^{* * *}$ & 1.8 & $0.01^{*}$ & - & - \\
\hline Crop $\times$ soil & 1 & 2.9 & $0.05 \mathrm{~ns}$ & 2.9 & $0.0006^{* * *}$ & 0.8 & $0.89 \mathrm{~ns}$ & 1.1 & $0.25 \mathrm{~ns}$ & 1.4 & $0.07 \mathrm{~ns}$ \\
\hline
\end{tabular}




\section{DISCUSSION}

The current study investigated the effect of plant compartment and soil properties on the diversity of bacterial communities associated with the rhizosphere and plant interior of wheat and canola plants grown in different soils in a growth chamber. Although our study was focused on bacterial microbiomes associated with crops, it is important to note that fungal communities (mycobiomes) also represent a significant fraction of the crop microbes and play essential roles in sustainable agricultural ecosystems (Pagano et al. 2017). Highthroughput sequencing analysis revealed that bacterial community structure of wheat and canola differed among the rhizosphere, root, and aboveground plant organs. The rhizosphere and roots of both

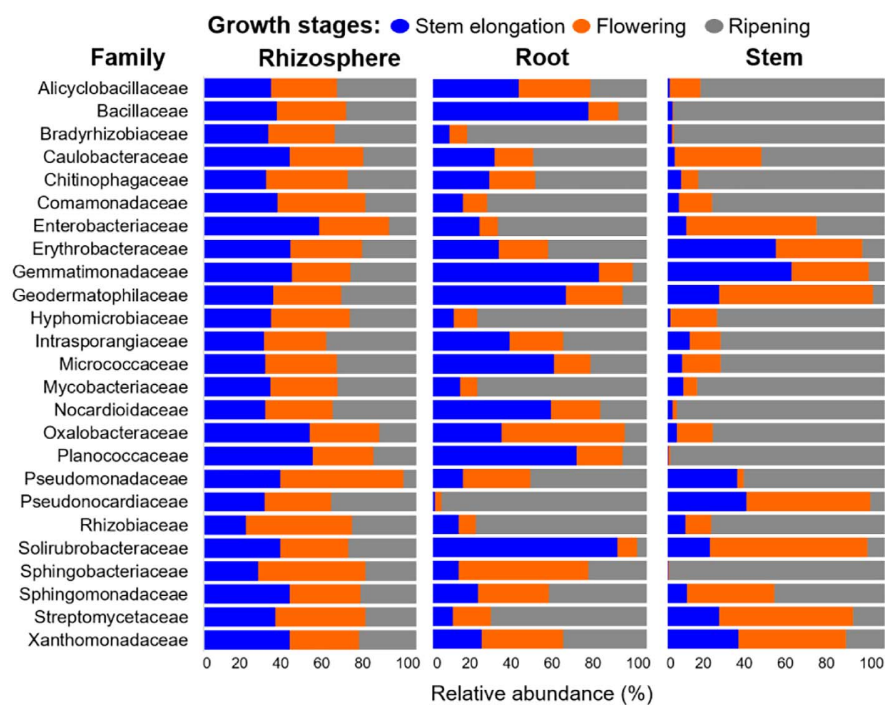

Fig. 3. Relative abundance of bacterial families ( $>0.5 \%$ abundant) associated with rhizosphere, roots, and stems of wheat harvested at stem elongation, flowering, and ripening. Relative abundance of bacterial families was averaged from plants grown in brown and black soils in Saskatchewan.

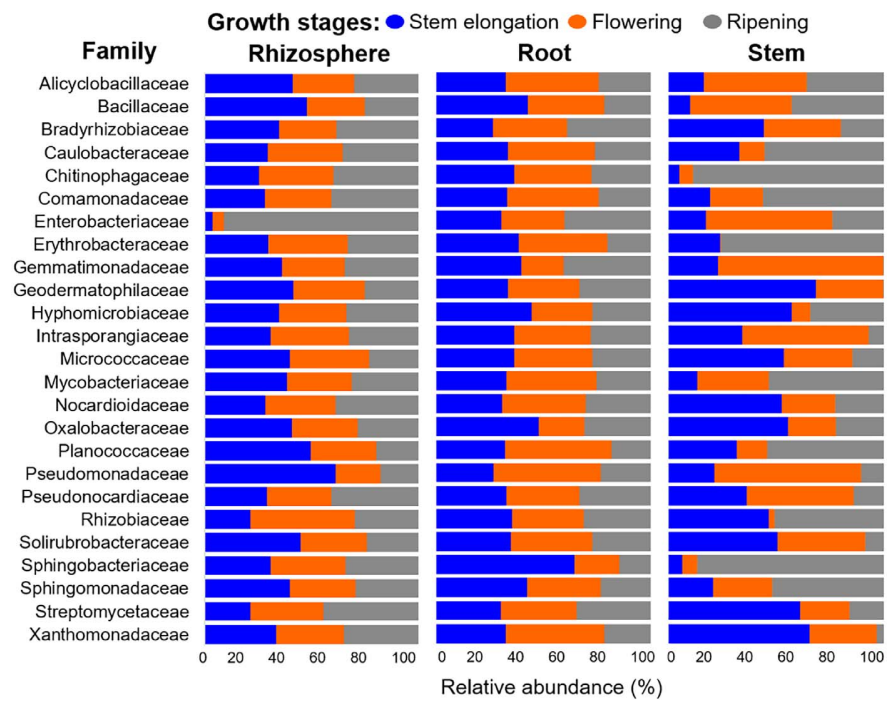

Fig. 4. Relative abundance of bacterial families (>0.5\% abundant) associated with rhizosphere, roots, and stems of canola harvested at stem elongation, flowering, and ripening. Relative abundance of bacterial families was averaged from plants grown in brown and black soils in Saskatchewan. crops exhibited a higher number of OTUs compared with the aboveground plant organs. These findings support the idea that endophytic bacterial communities are a subset of the rhizosphere microbiome (Bulgarelli et al. 2013; Edwards et al. 2015; Germida et al. 1998). The lower diversity and species richness of the bacterial communities associated with wheat and canola aboveground plant organs may be related to the low number of endophytes that are able to colonize aerial plants organs. In fact, Hallmann (2001) reported that bacterial endophytes have to overcome several plant morphological barriers and physiological limitations to establish in the stems, leaves, and seed.

Our analysis of bacterial community structure associated with wheat and canola indicated that rhizosphere communities were mainly influenced by soil characteristics, as compared with the effect of host crop or plant growth stages. Similarly, Simonin et al. (2020) also reported that soil characteristics had the largest influence on microbial taxa richness and in community structure of wheat grown in African and European soils. In addition, Garbeva et al. (2004) reported that soil properties have the ability to influence the bacterial communities not only in the bulk soil but also in microbial communities in the plant's rhizosphere. Rhizosphere bacteria associated with wheat and canola were characterized by the dominance of bacterial genera with known beneficial effects on these crops. For example, the genus Gemmatimonas was predominant in the rhizosphere of wheat and canola grown in both brown and black soils in the growth chamber, confirming our recent findings for field-grown canola and wheat grown in soils from the same locations (Cordero et al. 2020). Similarly, DeBruyn et al. (2011) and Gkarmiri et al. (2017) reported Gemmatimonas to be widely distributed in agricultural systems. These authors also reported a high relative abundance of members of the phylum Gemmatimonadetes associated with plants grown in soils with $\mathrm{pH}$ near neutrality, somewhat similar to the $\mathrm{pH}$ of the brown and black soils that were used in the current study. The rhizosphere of canola and wheat grown in brown soil exhibited a high abundance of the genera Solirubrobacter and Nocardioides, which have been previously detected in rhizosphere soils associated with wheat and canola (Donn et al. 2015; Monreal et al. 2018). Similar to the current investigation, Germida et al. (1998) and Germida and Siciliano (2001) also reported that Arthrobacter was detected in the rhizosphere of wheat, thus suggesting that Arthrobacter is among the most prevalent genera associated with the rhizosphere of wheat grown in Saskatchewan agricultural soils.

In the current study, root bacterial endophytes differed between wheat and canola, suggesting that each crop selected distinct bacterial communities. The selection of endophytic communities from the rhizosphere occurs during the root colonization as the host crop regulates the colonization and multiplication of specific bacterial taxa in the root interior. For example, Bulgarelli et al. (2013) reported that the release of root exudates in the rhizosphere has an important function for the selection of root microbiota by the crop. Several plant characteristics such as plant health, plant developmental stages, root morphology, and the presence of plant wounds also may affect the penetration of bacteria into the host plant roots (Gaiero et al. 2013; Garbeva et al. 2004). Furthermore, bacterial colonization within the plant tissues involve physiological traits of bacteria such as the production of lipopolysaccharides, the activity of flagella or pili, the secretion of cell-wall-degrading enzymes, and the degradation of plant-derived compounds (Compant et al. 2010). Boller and $\mathrm{He}$ (2009) also suggested that some bacterial endophytes may possess effective mechanisms to avoid detection by a plant's immune system and, thus, establish viable colonies inside the plant tissues. The root interior of wheat grown in potted soils in the current growthchamber study exhibited a high abundance of Stenotrophomonas, Streptomyces, and Variovorax. This confirms our previous and other 
researchers' observations for field-grown crops (Cordero et al. 2020; Lay et al. 2018) in Canadian prairie soils. These bacterial genera are reported to exert beneficial effects on wheat (Bertrand et al. 2001; Coombs and Franco 2003; Coombs et al. 2004; Conn and Franco 2004; Dal Bello et al. 2002; Germida and Siciliano 2001; Germida et al. 1998; Majeed et al. 2015; Mouloud et al. 2015; Rascovan et al. 2016; Ryan et al. 2009; Salantur et al. 2006; Singh and Jha 2017; Yin et al. 2013).

Surprisingly, our results revealed that neither crop species nor soil characteristics influenced the composition of bacterial endophytes associated with wheat or canola aboveground plant organs. In contrast to the root bacterial communities, endophytic bacteria associated with stem, leaf, and seed of wheat and canola exhibited similar bacterial community profiles among crops. This observation may suggest that bacterial communities associated with aboveground plant organs were less influenced by crop genotype in comparison with the rootassociated bacteria (Gdanetz and Trail 2017; Ofek-Lalzar et al. 2014; Peiffer et al. 2013). We also found that bacterial communities associated with wheat and canola were highly variable among stems, leaves, and seed. Hirano and Upper (2000) reported that microbial communities associated with the aboveground plant organs are influenced by several abiotic factors such as temperature, humidity, light irradiation, and access to nutrients, which are more fluctuating in the aboveground plant organs in comparison with the rhizosphere and root environments. However, our growth-chamber experiment environmental conditions were maintained constant, in contrast to field studies, where fluctuations in abiotic factors may occur. Therefore, a possible explanation for the diverse bacterial profiles detected in the aboveground organs is that, during colonization of aerial organs, bacteria have to overcome several morphological barriers as well as need to possess the physiological requirements to establish in different plant niches existing in stems, leaves, or seed (Compant et al. 2010). In addition, these bacterial endophytic communities colonizing aboveground organs may originate from different sources, including roots or phyllosphere (Frank et al. 2017).

As indicated by high-throughput sequencing analysis, the bacterial microbiome associated with wheat and canola at stem elongation, flowering, and ripening varied among plant growth stages. Earlier studies examining the influence of growth stages on the bacterial communities associated with the rhizosphere, roots, and leaves of wheat and canola also reported a shift on the bacterial communities among growth stages (Chen et al. 2019; Copeland et al. 2015; de Campos et al. 2013; Dunfield and Germida 2003; Farina et al. 2012; Gdanetz and Trail 2017; Smalla et al. 2001). Similar to the results reported by Donn et al. (2015) and Smalla et al. (2001), the diversity of rhizosphere bacteria associated with wheat and canola was higher at ripening and flowering stages, when compared with the stem elongation growth stage. Gdanetz and Trail (2017) reported that fluctuations in the diversity of rhizosphere bacteria at flowering and maturity could be explained by three possible factors, including (i) root surface increases high availability of habitats and resources in the rhizosphere, (ii) signaling between host plant and microorganisms that colonized the rhizosphere at earlier stages, and (iii) availability of complex metabolites released by mature plant roots. Additionally, bacterial changes during plant growth may be associated with the activation of the plant defense system that can limit the spread of certain bacterial groups (Marag and Suman 2018). Our results revealed that the influence of growth stage on the bacterial microbiome was more noticeable in the stem compared with rhizosphere and roots compartments. Moreover, the influence of growth stage on bacterial richness and diversity was more significant in wheat compared with canola. These findings suggest that the influence of growth stage on the bacterial microbiome of wheat and canola was crop and organ specific. Taxonomic analysis of the most abundant bacterial family in both crops revealed that bacterial distribution among growth stages was even in the rhizosphere and highly variable at the stem. However, the roots of wheat exhibited a high variation in bacterial family among stages whereas, in canola, the distribution was even. These changes in the relative abundance of bacterial families among growth stages suggest that crops may select specific bacterial taxa within different plant compartments at each plant growth stage. In fact, previous studies concluded that the presence of certain bacterial groups at specific growth stages was related to the different bacterial ecological strategies within the host crop. For example, at early plant stages, fast-growing and efficient carbondegrading bacteria (r-strategist) may colonize such rapidly changing environments as the young growing roots (Chiarini et al. 1998). In contrast, at mature stages, bacterial communities in the roots are reported to be dominated by $\mathrm{K}$-strategists (i.e., bacteria exhibiting low growth rates and high persistency, even under low nutrient availability) (Brimecombe et al. 2000; Chiarini et al. 1998). Similarly, a previous study by Marag and Suman (2018) reported that, in the aboveground organs, a declining of bacterial populations occurs at maturity stage, partially due to factors such as nutrient deficiency, water stress, and free radical formation.

In conclusion, this study provides information on the diversity of the bacterial microbiome associated with plant organs of wheat and canola grown in agricultural soils from Saskatchewan, Canada. Bacterial community structure in both crops exhibited distinct profiles between the rhizosphere, root, and aboveground plant organs. Rhizosphere bacteria associated with wheat and canola differed between plants grown in brown and black soils, suggesting that soil properties influenced the rhizosphere microbiome, whereas crop species influenced endophytic bacteria associated with the roots of the crops. In contrast, the aboveground plant organs exhibited a high variability among crops and soils, thus suggesting that additional factors, including physiological and morphological complexity and diverse bacterial colonization pathways of aboveground tissues, may have influenced the bacterial microbiome in stems, leaves, and seed. Additionally, plant growth stages also may influence the bacterial microbiome associated with the rhizosphere, root, and aboveground plant organs of wheat and canola. The influence of growth stages on the bacterial microbiome of wheat and canola was crop and organ specific. Overall, our results suggest that transmission and dynamics of bacteria in the rhizosphere, root, and aboveground plant organs of crops may be controlled by interactions between multiple factors. Bacterial communities associated with the rhizosphere are highly influenced by soil properties whereas root endophytes are mainly controlled by crop species. Aboveground communities are affected by both plant physiological process and habitat complexity within the plant tissues, which may influence the colonization and diversity of endophytic bacteria in stem, leaf, and seed.

\section{LITERATURE CITED}

Ali, S., Charles, T. C., and Glick, B. R. 2014. Amelioration of high salinity stress damage by plant growth-promoting bacterial endophytes that contain ACC deaminase. Plant Physiol. Biochem. 80:160-167.

Baudoin, E., Benizri, E., and Guckert, A. 2002. Impact of growth stage on the bacterial community structure along maize roots, as determined by metabolic and genetic fingerprinting. Appl. Soil Ecol. 19:135-145.

Berg, G., Grube, M., Schloter, M., and Smalla, K. 2014. Unraveling the plant microbiome: Looking back and future perspectives. Front. Microbiol. 5:148.

Bertrand, H., Nalin, R., Bally, R., and Cleyet-Marel, J. C. 2001. Isolation and identification of the most efficient plant growth-promoting bacteria associated with canola (Brassica napus). Biol. Fertil. Soils 33:152-156.

Boller, T., and He, S. Y. 2009. Innate immunity in plants: An arms race between pattern recognition receptors in plants and effectors in microbial pathogens. Science 324:742-744. 
Bright, M., and Bulgheresi, S. 2010. A complex journey: Transmission of microbial symbionts. Nat. Rev. Microbiol. 8:218-230.

Brimecombe, M. J., De Leij, F. A., and Lynch, J. M. 2000. The effect of root exudates on rhizosphere microbial populations. Pages 95-140 in: The Rhizosphere, Biochemistry and Organic Substances at the Soil-Plant Interface. R. Pinton, Z. Varini, and P. Nannipieri, eds. CRC Press, Boca Raton, FL, U.S.A.

Bulgarelli, D., Schlaeppi, K., Spaepen, S., Ver Loren van Themaat, E., and Schulze-Lefert, P. 2013. Structure and functions of the bacterial microbiota of plants. Annu. Rev. Plant Biol. 64:807-838.

Caporaso, J. G., Lauber, C. L., Walters, W. A., Berg-Lyons, D., Lozupone, C. A., Turnbaugh, P. J., Fierer, N., and Knight, R. 2010. Global patterns of 16S rRNA diversity at a depth of millions of sequences per sample. Proc. Natl. Acad. Sci. U.S.A. 108:4516-4522.

Chen, S., Waghmode, T. R., Sun, R., Kuramae, E. E., Hu, C., and Liu, B. 2019. Root-associated microbiomes of wheat under the combined effect of plant development and nitrogen fertilization. Microbiome 7:136.

Chiarini, L., Bevivino, A., Dalmastri, C., Nacamulli, C., and Tabacchioni, S. 1998. Influence of plant development, cultivar and soil type on microbial colonization of maize roots. Appl. Soil Ecol. 8:11-18.

Compant, S., Clément, C., and Sessitsch, A. 2010. Plant growth promoting bacteria in the rhizo- and endosphere of plants: Their role, colonization, mechanisms involved and prospects for utilization. Soil Biol. Biochem. 42: 669-678.

Conn, V. M., and Franco, C. M. 2004. Analysis of the endophytic actinobacterial population in the roots of wheat (Triticum aestivum L.) by terminal restriction fragment length polymorphism and sequencing of $16 \mathrm{~S}$ rRNA clones. Appl. Environ. Microbiol. 70:1787-1794.

Coombs, J. T., and Franco, C. M. 2003. Visualization of an endophytic Streptomyces species in wheat seed. Appl. Environ. Microbiol. 69:4260-4262.

Coombs, J. T., Michelsen, P. P., and Franco, C. M. 2004. Evaluation of endophytic actinobacteria as antagonists of Gaeumannomyces graminis var. tritici in wheat. Biol. Control 29:359-366.

Copeland, J. K., Yuan, L., Layeghifard, M., Wang, P. W., and Guttman, D. S. 2015. Seasonal community succession of the phyllosphere microbiome. Mol. Plant-Microbe Interact. 28:274-285.

Cordero, J., de Freitas, J. R., and Germida, J. J. 2020. Bacterial microbiome associated with the rhizosphere and root interior of crops in Saskatchewan, Canada. Can. J. Microbiol. 66:71-85.

Dal Bello, G. M., Monaco, C. I., and Simon, M. R. 2002. Biological control of seedling blight of wheat caused by Fusarium graminearum with beneficial rhizosphere microorganisms. World J. Microbiol. Biotechnol. 18:627-636.

DeBruyn, J. M., Nixon, L. T., Fawaz, M. N., Johnson, A. M., and Radosevich, M. 2011. Global biogeography and quantitative seasonal dynamics of Gemmatimonadetes in soil. Appl. Environ. Microbiol. 77:6295-6300.

de Campos, S. B., Youn, J. W., Farina, R., Jaenicke, S., Jünemann, S., Szczepanowski, R., and Passaglia, L. M. 2013. Changes in root bacterial communities associated to two different development stages of canola (Brassica napus L. var oleifera) evaluated through next-generation sequencing technology. Microb. Ecol. 65:593-601.

Donn, S., Kirkegaard, J. A., Perera, G., Richardson, A. E., and Watt, M. 2015. Evolution of bacterial communities in the wheat crop rhizosphere. Environ. Microbiol. 17:610-621.

Dunfield, K. E., and Germida, J. J. 2003. Seasonal changes in the rhizosphere microbial communities associated with field-grown genetically modified canola (Brassica napus). Appl. Environ. Microbiol. 69:7310-7318.

Edwards, J., Johnson, C., Santos-Medellín, C., Lurie, E., Podishetty, N. K., Bhatnagar, S., Eisen, J. A., and Sundaresan, V. 2015. Structure, variation, and assembly of the root associated microbiomes of rice. Proc. Natl. Acad. Sci. U.S.A. 112:E911-E920.

Edwards, J. E., Huws, S. A., Kim, E. J., and Kingston-Smith, A. H. 2007. Characterization of the dynamics of initial bacterial colonization of nonconserved forage in the bovine rumen. FEMS Microbiol. Ecol. 62: 323-335.

el Zahar Haichar, F., Marol, C., Berge, O., Rangel-Castro, J. I., Prosser, J. I., Balesdent, J., and Achouak, W. 2008. Plant host habitat and root exudates shape soil bacterial community structure. ISME J. 2:1221-1230.

Farina, R., Beneduzi, A., Ambrosini, A., de Campos, S. B., Lisboa, B. B., Wendisch, V., and Passaglia, L. M. 2012. Diversity of plant growthpromoting rhizobacteria communities associated with the stages of canola growth. Appl. Soil Ecol. 55:44-52.

Frank, A. C., Saldierna Guzmán, J. P., and Shay, J. E. 2017. Transmission of bacterial endophytes. Microorganisms 5:70.
Gaiero, J. R., McCall, C. A., Thompson, K. A., Day, N. J., Best, A. S., and Dunfield, K. E. 2013. Inside the root microbiome: Bacterial root endophytes and plant growth promotion. Am. J. Bot. 100:1738-1750.

Garbeva, P. V., Van Veen, J. A., and Van Elsas, J. D. 2004. Microbial diversity in soil: Selection of microbial populations by plant and soil type and implications for disease suppressiveness. Annu. Rev. Phytopathol. 42:243-270.

Gdanetz, K., and Trail, F. 2017. The wheat microbiome under four management strategies, and potential for endophytes in disease protection. Phytobiomes. 1:158-168.

Germida, J., and Siciliano, S. 2001. Taxonomic diversity of bacteria associated with the roots of modern, recent and ancient wheat cultivars. Biol. Fertil. Soils 33:410-415.

Germida, J. J., Siciliano, S. D., De Freitas, J. R., and Seib, A. M. 1998. Diversity of root-associated bacteria associated with field-grown canola (Brassica napus L.) and wheat (Triticum aestivum L.). FEMS Microbiol. Ecol. 26:43-50.

Gkarmiri, K., Mahmood, S., Ekblad, A., Alström, S., Högberg, N., and Finlay, R. 2017. Identifying the active microbiome associated with roots and rhizosphere soil of oilseed rape. Appl. Environ. Microbiol. 83:e01938-17.

Hallmann, J. 2001. Plant interactions with endophytic bacteria. Pages 87-119 in: Biotic Interactions in Plant-Pathogen Associations. M. J. Jeger and N. J. Spence, eds. CABI Publishing, New York, NY, U.S.A.

Hardoim, P. R., Hardoim, C. C., Van Overbeek, L. S., and Van Elsas, J. D. 2012. Dynamics of seed-borne rice endophytes on early plant growth stages. PLoS One 7:e30438.

Hardoim, P. R., van Overbeek, L. S., and van Elsas, J. D. 2008. Properties of bacterial endophytes and their proposed role in plant growth. Trends Microbiol. 16:463-471.

Hirano, S. S., and Upper, C. D. 2000. Bacteria in the leaf ecosystem with emphasis on Pseudomonas syringae-a pathogen, ice nucleus, and epiphyte. Microbiol. Mol. Biol. Rev. 64:624-653.

Houlden, A., Timms-Wilson, T. M., Day, M. J., and Bailey, M. J. 2008. Influence of plant developmental stage on microbial community structure and activity in the rhizosphere of three field crops. FEMS Microbiol. Ecol. 65:193-201.

Kandel, S. L., Joubert, P. M., and Doty, S. L. 2017. Bacterial endophyte colonization and distribution within plants. Microorganisms 5:77-103.

Kozich, J. J., Westcott, S. L., Baxter, N. T., Highlander, S. K., and Schloss, P. D. 2013. Development of a dual-index sequencing strategy and curation pipeline for analyzing amplicon sequence data on the MiSeq Illumina sequencing platform. Appl. Environ. Microbiol. 79:5112-5120.

Lancashire, P. D., Bleiholder, H., Boom, T. V. D., Langelüddeke, P., Stauss, R., Weber, E., and Witzenberger, A. 1991. A uniform decimal code for growth stages of crops and weeds. Ann. Appl. Biol. 119:561-601.

Lay, C. Y., Bell, T. H., Hamel, C., Harker, K. N., Mohr, R., Greer, C. W., Yergeau, E., and St-Arnaud, M. 2018. Canola root-associated microbiomes in the Canadian Prairies. Front. Microbiol. 9:1188.

Love, M. I., Huber, W., and Anders, S. 2014. Moderated estimation of fold change and dispersion for RNA-seq data with DESeq2. Genome Biol. 15:550.

Majeed, A., Abbasi, M. K., Hameed, S., Imran, A., and Rahim, N. 2015. Isolation and characterization of plant growth-promoting rhizobacteria from wheat rhizosphere and their effect on plant growth promotion. Front. Microbiol. 6:198.

Marag, P. S., and Suman, A. 2018. Growth stage and tissue specific colonization of endophytic bacteria having plant growth promoting traits in hybrid and composite maize (Zea mays L.). Microbiol. Res. 214:101-113.

McCune, B., and Grace, J. B. 2002. Analysis of Ecological Communities. MjM Software, Gleneden Beach, OR, U.S.A.

Micallef, S. A., Shiaris, M. P., and Colón-Carmona, A. 2009. Influence of Arabidopsis thaliana accessions on rhizobacterial communities and natural variation in root exudates. J. Exp. Bot. 60:1729-1742.

Monreal, C. M., Zhang, J., Koziel, S., Vidmar, J., González, M., Matus, F., and Etcheverria, P. 2018. Bacterial community structure associated with the addition of nitrogen and the dynamics of soluble carbon in the rhizosphere of canola (Brassica napus) grown in a Podzol. Rhizosphere 5:16-25.

Mougel, C., Offre, P., Ranjard, L., Corberand, T., Gamalero, E., Robin, C., and Lemanceau, P. 2006. Dynamic of the genetic structure of bacterial and fungal communities at different developmental stages of Medicago truncatula Gaertn. cv. Jemalong line J5. New Phytol. 170:165-175.

Mouloud, G., Samir, M., Hani, B., and Daoud, H. 2015. Biocontrol of wheat Fusarium head blight (FHB) by Streptomyces spp. isolated from the 
rhizosphere of Astragalus gombo Coss. and Dur. and Ononis angustissima Lam. Am.-Eurasian J. Agric. Environ. Sci. 15:2499-2511.

Nelson, E. B. 2004. Microbial dynamics and interactions in the spermosphere. Annu. Rev. Phytopathol. 42:271-309.

Ofek-Lalzar, M., Sela, N., Goldman-Voronov, M., Green, S. J., Hadar, Y., and Minz, D. 2014. Niche and host-associated functional signatures of the root surface microbiome. Nat. Commun. 5:4950.

Pagano, M. C., Correa, E. J. A., Duarte, N. F., Yelikbayev, B., O’Donovan, A., and Gupta, V. K. 2017. Advances in eco-efficient agriculture: The plant-soil mycobiome. Agriculture 7:14-26.

Peiffer, J. A., Spor, A., Koren, O., Jin, Z., Tringe, S. G., Dangl, J. L., and Ley, R. E. 2013. Diversity and heritability of the maize rhizosphere microbiome under field conditions. Proc. Natl. Acad. Sci. U.S.A. 110:6548-6553.

R Core Team. 2021. R: A language and environment for statistical computing. R Foundation for Statistical Computing, Vienna, Austria. https://www.R-project.org/

Rascovan, N., Carbonetto, B., Perrig, D., Díaz, M., Canciani, W., Abalo, M., and Vazquez, M. P. 2016. Integrated analysis of root microbiomes of soybean and wheat from agricultural fields. Sci. Rep. 6:28084.

Reinhold-Hurek, B., and Hurek, T. 2011. Living inside plants: Bacterial endophytes. Curr. Opin. Plant Biol. 14:435-443.

Ryan, R. P., Monchy, S., Cardinale, M., Taghavi, S., Crossman, L., Avison, M. B., and Dow, J. M. 2009. The versatility and adaptation of bacteria from the genus Stenotrophomonas. Nat. Rev. Microbiol. 7:514-525.

Salantur, A., Ozturk, A., and Akten, S. 2006. Growth and yield response of spring wheat (Triticum aestivum L.) to inoculation with rhizobacteria. Plant Soil Environ. 52:111-118.

Sessitsch, A., Reiter, B., Pfeifer, U., and Wilhelm, E. 2002. Cultivationindependent population analysis of bacterial endophytes in three potato varieties based on eubacterial and Actinomycetes-specific PCR of $16 \mathrm{~S}$ rRNA genes. FEMS Microbiol. Ecol. 39:23-32.
Siciliano, S. D., and Germida, J. J. 1999. Taxonomic diversity of bacteria associated with the roots of field-grown transgenic Brassica napus cv. Quest, compared to the non-transgenic B. napus cv. Excel and B. rapa cv. Parkland. FEMS Microbiol. Ecol. 29:263-272.

Simonin, M., Dasilva, C., Terzi, V., Ngonkeu, E. L., Diouf, D., Kane, A., Bena, G., and Moulin, L. 2020. Influence of plant genotype and soil on the wheat rhizosphere microbiome: Evidences for a core microbiome across eight African and European soils. FEMS Microbiol. Ecol. 96:fiaa067.

Singh, R. P., and Jha, P. N. 2017. The PGPR Stenotrophomonas maltophilia SBP-9 augments resistance against biotic and abiotic stress in wheat plants. Front. Microbiol. 8:1945.

Smalla, K., Wieland, G., Buchner, A., Zock, A., Parzy, J., Kaiser, S., and Berg, G. 2001. Bulk and rhizosphere soil bacterial communities studied by denaturing gradient gel electrophoresis: Plant-dependent enrichment and seasonal shifts revealed. Appl. Environ. Microbiol. 67:4742-4751.

Statistics Canada. 2021. Estimated areas, yield, production, average farm price and total farm value of principal field crops, in metric and imperial units. Table 32-10-0359-01. https://www150.statcan.gc.ca/t1/tbl1/en/tv.action? pid=3210035901

Turner, T. R., Ramakrishnan, K., Walshaw, J., Heavens, D., Alston, M., Swarbreck, D., and Poole, P. S. 2013. Comparative metatranscriptomics reveals kingdom level changes in the rhizosphere microbiome of plants. ISME J. 7:2248-2258.

Vincent, J. M. 1970. A Manual for the Practical Study of Root-Nodule Bacteria. Blackwell Scientific, Oxford, England.

Yin, C., Hulbert, S. H., Schroeder, K. L., Mavrodi, O., Mavrodi, D., Dhingra, A., and Paulitz, T. C. 2013. The role of bacterial communities in the natural suppression of Rhizoctonia bare patch of wheat (Triticum aestivum L.). Appl. Environ. Microbiol. 79:7428-7438. 